\title{
Fortalecimiento de las Competencias de Aprendizaje de Geometría mediante B-Learning
}

Strengthening of Geometry Learning Competencies through B-Learning.

Fabinton Sotelo ${ }^{1}$ Mario Solarte ${ }^{2}$,Wilson Jordan ${ }^{3}$, Yerson Erazo ${ }^{4}$

Fecha de recepción: 12 de agosto de 2019

Fecha de aceptación: 25 de octubre de 2019

(C) 2019. Universidad de Cordoba. Este es un artículo de acceso abierto distribuido bajo los términos de la licencia Creative Commons Attribution License, que permite el uso ilimitado, distribución y reproducción en cualquier medio, siempre que el autor original y la fuente se acreditan.

\section{RESUMEN}

Una de las debilidades frecuentes encontradas en el aprendizaje de los estudiantes de la educación media Colombiana se da en el área de las matemáticas, incluyendo el álgebra, trigonométrica, calculo y geometría. Esta investigación busca fortalecer la adquisición de competencias del área de la geometría del grado noveno, en un colegio de la ciudad de Popayán (Colombia), con aproximadamente 70 estudiantes. Haciendo uso del sistema de gestión de aprendizaje (LMS) dotLRN, Geogebra y un marco de referencia que integra esta herramienta al LMS. Se capacita al docente y estudiantes para hacer uso de estos servicios TIC, de tal forma se logren involucrar en el proceso educativo en temáticas como: Construcción de polígono, calculo de perímetro y calculo de área.

Se propone un caso de estudio donde se proponen las temáticas en dos cursos, con contextos educativos diferentes, 9A apoyado en b-learning y 9B de forma convencional; al final se comparan resultados académicos.

PALABRAS CLAVE: b-learning, TIC, dotLRN, LMS, Geogebra, educación media, geometría, competencias matemáticas.

\section{ABSTRACT}

One of the frequent weaknesses found in the learning of Colombian high school students occurs in the

${ }^{1} \mathrm{PhD}(\mathrm{c})$ Ingeniería Telemática. Estudiante doctorado ingeniería telemática, Docente ingeniería de sistemas Fundación Universitaria de Popayán. Colombia. fabinton. sotelo@unicacuca.edu.co.

${ }^{2} \mathrm{PhD}(\mathrm{c})$ Ingeniería Telemática. Docente Ingeniería telemática Universidad del Cauca . Colombia. msolarte@unicauca.edu.co.

3 Ingeniería de sistemas (c). Estudiante ingeniería de sistemas, Fundación Universitaria de Popayán. Colombia, wjordananaya@hotmail.com

${ }^{4}$ Ingeniería de sistemas (c). Estudiante ingeniería de sistemas, Fundación Universitaria de Popayán. Colombia. jersonerazo@gmail.com 
area of mathematics, including algebra, trigonometric, calculus and geometry. This research seeks to strengthen the acquisition of skills in the area of geometry in the ninth grade, in a school in the city of Popayán (Colombia), with approximately 70 students. Using the dotLRN learning management system (LMS), Geogebra and a framework that integrates this tool into the LMS. The teacher and students are trained to use these ICT services, in such a way that they are involved in the educational process in topics such as: Construction of a polygon, calculation of perimeter and calculation of area.

A case study is proposed where the themes are proposed in two courses, with different educational contexts, 9A supported by b-learning and 9B in a conventional way; at the end academic results are compared.

\section{KEYWORDS:}

Inverted class, meaningful learning, Anki, contest in class.

\section{INTRODUCCIÓN}

El Ministerio de Educación Nacional Colombiano define los Estándares Básicos de competencia como Criterio que permite juzgar si un estudiante, cumple con calidad lo que se espera que aprendan (saber y saber hacer).

Geogebra: Software educativo para matemáticas, geometría, álgebra, hoja de cálculo, gráficos, estadística, cálculo, ecuaciones, crear gráficas, trabajar con derivadas e integrales, Disponible en versión Web y local.

Basados en el desarrollo del presente artículo se platea el uso de las TIC como una herramienta de mediación pedagógica para la obtención de las competencias en el área de conocimiento que se aplica, puesto que reportes estadísticos de de las pruebas de estado Saber11 hacen notar que una de las áreas con más mortandad académica es el área de las matemáticas, la cual incluye asignaturas como aritmética, álgebra, geometría trigonométrica y calculo. Basados en este preocupante dato el presente trabajo evidencia una ardua investigación para brindar una posible alternativa apoyada en TIC a docentes y estudiantes de mejorar los resultados que se obtienen en las competencias de aprendizaje que se evalúan en el sistema educativo. Con la disposición del docente de geometría y de informática para involucrar a los estudiantes en el mundo del uso de herramientas software diseñadas para apoyar sistemas educativos virtuales ahora llevado a las aulas de la clase tradicional. Así se refleja como un LMS, Un marco de referencia de integración de recursos web al LMS y una gran y avanzada herramienta para el uso del desarrollo de competencias de aprendizaje de geometría como lo es Geogebra contribuyen a dinamizar una clase, configurando así un ambiente educativo b-learning, donde las TIC juegan el papel de dinamizar una clase tradicional.
Este artículo da a conocer el primer resultado del desarrollo de trabajo de grado de ingeniería de sistemas enmarcado en la tesis doctoral "Marco de Referencia para la integración de estrategias usadas en los MOOC en Educación Media" donde se aplica en un caso de estudio real de contextos educativos el framework de referencia expuesto en [5] y evaluado en [6] para fortalecer la adquisición de competencias en las temáticas especificas mencionadas en la asignatura de geometría.

La Institución Educativa Rafael Pombo donde se investiga el impacto de este trabajo, tiene como misión "formar niños, niñas y jóvenes en valores, propositivos, críticos, emprendedores, apoyados en la tecnología y procesos investigativos para alcanzar una educación integral que les permita generar su proyecto de vida y contribuyan al cambio social. Tiene como visión ser reconocida por su prestigio y por su impacto en la formación de sus educandos y egresados como gestores de cambio social" [2].

La institución se encuentra en el centro histórico de la ciudad de Popayán cuenta aproximadamente con 570 estudiantes desde el grado cero a undécimo, las edades oscilan entre los 4 a 19 años, son de estrato socio-económico cero, uno y dos. Por el sondeo que se ha realizado en una encuesta el acceso a la tecnología es muy restringido para aproximadamente el $60 \%$ de su población [8]. De la misma forma se hace un estudio predio del uso que se hace de las TIC en los procesos educativos y se detecta que las únicas áreas que recurren a esta es la de Tecnología e Informática e inglés, las demás no hacen uso o lo hacen de manera muy exporadica sin una metodología que involucre capacitación de docente y estudiantes.

Bajo la descripción del anterior contexto notamos que la institución educativa es propicia para llevar a cabo el caso de estudio que se plantea aquí, puesto que se pretende romper una brecha tecnología con el uso del marco de referencia 
para la adquisición de la competencia requerida, dando así la oportunidad a docentes y estudiantes el uso masivo a recursos software en bien de mejorar el rendimiento individual académico de los aprendices.

La gestión del aprendizaje electrónico - e-learning frecuentemente se soportan por las plataformas de gestión de aprendizaje - LMS [2]. Un LMS es un software que apoya los procesos de aprendizaje a través de herramientas didácticas y de comunicación entre estudiantes y profesores, estas pueden ser usadas para soportar cursos presenciales, semipresenciales e incluso a distancia [3]; para este caso en particular se aplica el concepto de bleanding learning (b-learning) [4] para la cual las TIC apoyan un proceso educativo convencional donde la prespecialidad del estudiante y docente no se remplazan sino que cuentan con un nuevo elemento tecnológico en el aula de clase.

En cuanto a los sistemas de gestión de aprendizaje existen diversas plataformas, por ejemplo: Moodle [9] y dotLRN2 [10], dos de los LMS open source más utilizados. Aunque la elección de una u otra plataforma corresponde exclusivamente a los requerimientos de cada institución o proyecto. dotLRN ofrece un gran conjunto de ventajas dado su arquitectura y su enfoque empresarial [11].

Como tal el uso de contenidos y servicios web educativos en entornos LMS es costoso, pues su desarrollo implica el conocimiento de profesionales expertos en el área de la pedagogía y informática, esto dificulta la difusión de estas valiosas herramientas. En este contexto la integración de recursos Web existentes dentro de un LMS ofrece innumerables ventajas: i) permite el uso gratuito de una gran cantidad de contenidos y servicios e-learning educativos disponible en internet. ii) Potencializa la cantidad y calidad de los servicios educativos de un LMS puesto que abre la posibilidad de interactuar con vídeos, imágenes, texto, simuladores, multimedia, sonido, herramientas didácticas, etc. iii) Disminuye la distracción de los estudiantes, puesto que si los recursos están integrados al LMS, no es necesario que el estudiante salga de la plataforma para acceder a otros sitios web. Esto último favorece la concentración y el aprovechamiento del tiempo. Respecto a la integración en mención es posible encontrar diversos trabajos sobre la integración de recursos web como servicios educativos en diferentes LMS; pero existen muy pocos trabajos que aborden la plataforma dotLRN; Esta integración plantea grandes retos debido a la arquitectura y el lenguaje de programación particulares [12].Este problema de investigación ha sido abordado recientemente, y los resultados parciales se documentan en [13] donde se presenta una revisión detallada sobre las aproximaciones existentes para integrar recursos Web en dotLRN; así mismo en [5] se describe un marco de referencia para incorporar estos recursos Web en al LMS.

El framework de referencia propuesto en [5] ofrece unos lineamientos arquitectónicos y de integración que definen el proceso que debe seguirse para integrar recursos Web en dotLRN. Estos lineamientos ofrecen una hoja de ruta estándar hasta ahora inexistentes que permiten que cualquier docente, administrador o gestor de contenidos pueda utilizar materiales disponibles de en internet para soportar sus clases usando dotLRN e innove en sus procesos de aprendizaje y enseñanza. De este modo este artículo se enfoca en hacer uso de este aporte de Maestría en Ingeniería Telemática en un contexto diferente (zona urbana) al que ya se había usado (zonas rurales) y en el fortalecimiento de la adquisición de los estándares básicos de competencias en las temáticas ya mencionada.

El presente artículo describe los lineamientos del framework de referencia en un colegio de la zona urbana. El resto de este artículo se organiza de la siguiente forma: La sección 3 Implementación de la integración de geogebra al LMS, y en la 4, se exponen las conclusiones.

\section{Framework para la Integración de Geogebra al LMS dotLRN}

\subsection{Sistema de Gestión de Aprendizaje dotLRN.}

dotLRN se centra en facilitar la comunicación entre los actores en la experiencia de aprendizaje. Así desde el registro se da la opción al estudiante de compartir documentos, contar con una administración de usuarios y gestión de comunidades. Dentro los pocos trabajos que se documentan acordes a esta investigación, se detecta que la plataforma ofrece algunas formas para interactuar con agentes externos a su propio núcleo de módulos quedando abierta la posibilidad de interacción con otros LMS y recursos Web externos a ella [14] [15] y [16]. El marco de referencia para la integración de recursos web como servicios de e-learning en dotLRN propuesto en [5], define los requisitos y 
lineamientos arquitectónicos para la integración de recursos web dentro de dotLRN.

dotLRN presenta los siguientes servicios a los usuarios en su entorno virtual de aprendizaje[17] [18] [19]: Calendario, Blog, Documentos, Wiki, Creación y gestión de comunidades, Repositorios de contenido, Cuestionarios y Evaluación, Foros, [13], Lista de miembros, FAQs, Internacionalización i18n, Autenticación [20] [21], Tareas, Seguimiento de usuarios, Sistema de repositorio de objetos de aprendizaje - LORS, Álbum de Fotos, WebDAV, ECommerce, WysiWyg - editor Web, WimpyPoint, AJAX [22] [23], Templating.

\subsection{Recursos web}

Para este contexto se define recurso web como elementos que estén identificados por una URI (identificador uniforme de recurso) alojada en Internet y accedida mediante una versión del protocolo HTTP según el ISBD ER (Estándar International de Descripción Bibliográfica) y la W3C (consorcio de la red alrededor del mundo) [24]. Así mismo se asume un servicio de e-learning, como recursos informáticos bajo una arquitectura de servicios; tomando como definición de servicio propuesto en [25] y [26].

Los recursos web en su gran mayoría son accedidos usando el protocolo HTTP, lo que permite trabajar con recursos hechos en flash, java, javascript, html, html5, Web 2.0, entre otros. Debido a que en la Web se pueden encontrar muchos recursos HTTP de carácter educativo o al menos que presten un servicio de este tipo es necesario establecer unos requisitos mínimos que garanticen su integración con dotLRN tal como se expone en [5]; Por otra parte, el marco de referencia define además los lineamientos arquitectónicos para integrar recursos Web que presten servicios e-learning en el LMS dotLRN teniendo en cuenta su arquitectura.

\subsection{Lineamientos del framewor de integración.}

Es de mencionar que en [6] se ha validado el marco de referencia para la integración de recursos web educativos en dotLRN, en síntesis este menciona 2 conjuntos de aspectos, estos son: Requisitos del recursos web para ser integrado en dotLRN descritos en la sección 3,1 y los Lineamientos arquitectónicos para la integración de recursos eeb en DotLRN descritos en 3.2.

\section{Experiencia de Aplicación en ClaseImplementación del framework para integrar recursos geogebra}

A continuación se describe el desarrollo de la aplicación en dotLRN que permita integrar Geogebra, se sigue los criterios y lineamientos arquitectónicos propuestos en el framework. Para efectos de esta implementación se tiene creado un curso virtual "Geometria", un docente y los estudiantes en la plataforma.

\subsection{Verificación de requisitos.}

En la tabla 1 se realiza la verificación de requisitos de los servicios a integrar, puesto que es lo primero que se debe cumplir para cumplir con el marco de referencia:

Tabla 1. Verificación de criterios de integración para geogebra

\begin{tabular}{|c|l|c|}
\hline No & \multicolumn{1}{|c|}{ Criterio } & Cumplei(Si/No) \\
\hline 1 & Identificación de la URL del recurso & $\mathrm{Si}$ \\
\hline 2 & Verificación del tamaño de visualización & $\mathrm{Si}$ \\
\hline 3 & Funcionalidades compatibles con HTTP & $\mathrm{Si}$ \\
\hline 4 & Compatibilidad con la mayoría de navegadores & $\mathrm{Si}$ \\
\hline 5 & El recurso apoya un proceso de aprendizaje S & $\mathrm{Si}$ \\
\hline 6 & Posee una URL & \multicolumn{1}{|c|}{} \\
\hline
\end{tabular}

Como se evidencia Geogebra cumple con los requisitos mínimos para ser integrado al LMS. 


\subsection{Sistema de Gestión de Aprendizaje dotLRN.}

A continuación se describe los lineamientos que se siguieron para la integración Geogebra en dotLRN: 1. Se crea el paquete por medio del Package Manager (http://localhost:9000/acs-admin/apm): El nombre del paquete en estudio es "Geometría": en donde posteriormente se integra Geogebra. 2. Programar el paquete para hacer referencia a los recurso web a integrar teniendo en cuenta los criterios de integración: Aquí se crean los archivos necesarios para administrar los recursos de Geogebra, en el directorio del paquete "recursoGeometria". 3. Creación de los directorios del portlet y el applet con ayuda del script (código para ejecutar) de Nima Mazloumi: Se siguieron las instrucciones del script teniendo en cuenta la información del paquete creado. Para no copiar los directorios de applet y portlet generados al directorio de paquetes de (/usr/share/packages) se copió el script run.tcl junto con las carpetas de Nima Mazloumi directamente en la carpeta / usr/share/dotlrn/packages teniendo en cuenta los privilegios de lectura, escritura y propietario como los demás paquetes. Una vez ejecutado el script run.tcl y diligenciado los datos que se solicitan, ya se obtienen los tres directorios de la aplicación (recurso-Geometria, recursoGeomeria-portlet y dotlrn-recurso-Geometria). 4. Se programa el portlet para describir el recurso web Geogebra y vincularlo al paquete y desarrollar su administración. En el caso en estudio se crea dentro de la ruta/usr/share/dotlrn/packages/ recurso-Geometria/www, el archivo index.adp el cual contiene el código para redireccionar al recurso web. 5. Como lo sugiere el marco de referencia se programa el applet solo si es necesario. Para nuestro caso no se hace necesario puesto que el applet generado cumple con los requisitos trabajados en el portlet. 6 . Se instala el applet y el portlet, por medio de la herramienta de instalación de software de dotLRN en la opción Install from Local - Service - selección del applet de Recursos Geometria. 7. Finalmente se verifica que la aplicación esté disponible para ser agregada en el curso desde la opción Administrar applets en el portal Admin del curso por el docente encargado. En la figura 1 se muestra el applet "Recursos Geometría" el cual ya puede ser llamado desde cualquier curso virtual que lo requiera. Observe figura 1.

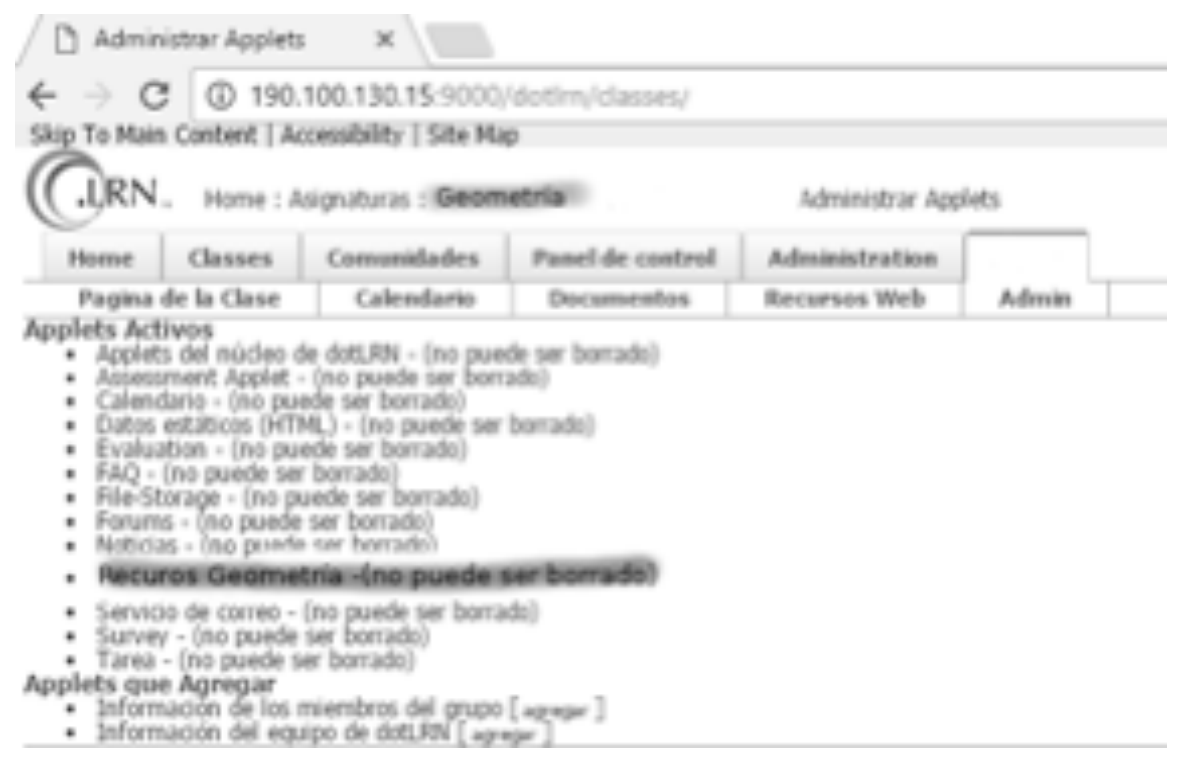

Figura 1. Applet de Recursos de Geometría

\subsection{Verificación de la implementación del marco de referencia.}

1. Luego de la implementación se verifica el rendimiento y conectividad de la plataforma en el hardware instalado (servidor) mediante el acceso de clientes (docente y estudiante).

2. Se consigue listar el applet entre los applets disponibles en la herramienta de administración de applets del docente. 
3. Una vez disponible el applet se insertar a la página principal del curso el portlet de Recurso Geogebra que maneja el recurso web educativo. 4. Automáticamente se inserta el portlet de administración del paquete en la página de administración del curso, donde se lista Geogebra ya integrados al LMS. Todos los recursos web integrados a la plataforma se ven como se muestra en la figura dos:

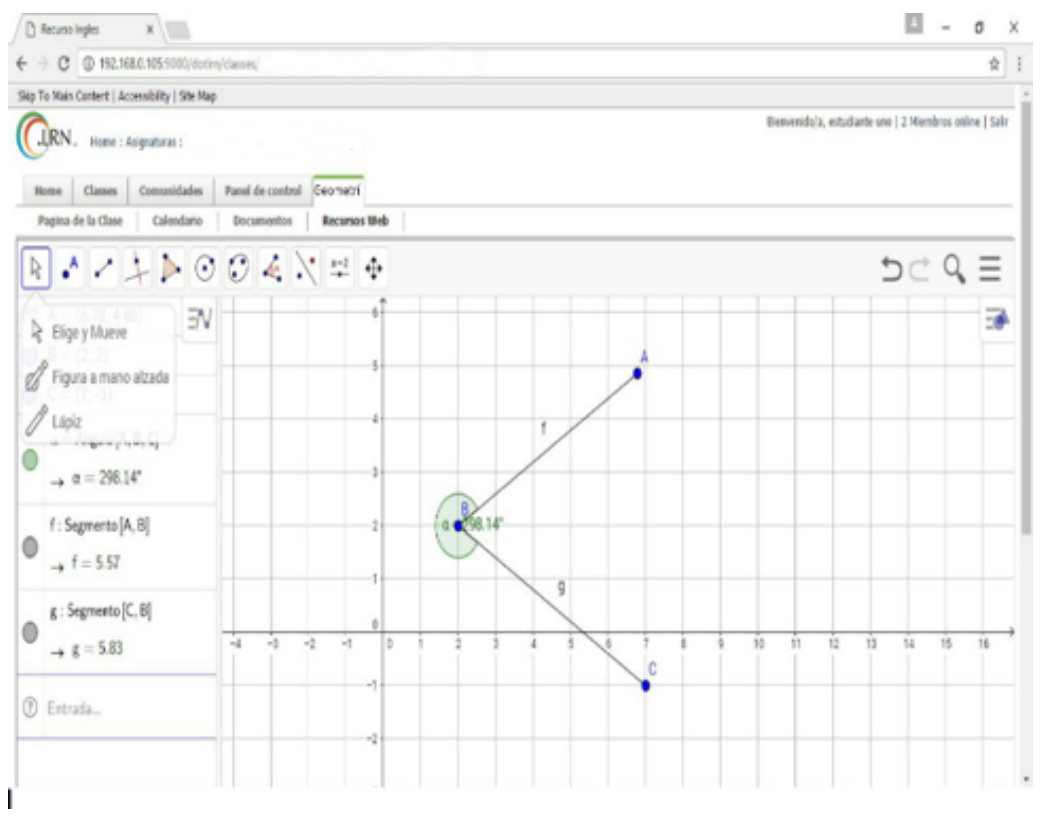

Figura 2. Geogebra integrado a dotLRN

\section{Framework para la Integración de Geogebra al LMS dotLRN}

En esta sección se describe el caso de estudio que se realizo, este se lleva a cabo mediante una serie de reuniones con las personas involucradas. Cada encuentro tiene como fin contextualizar y enseñar a los usuarios el uso de las plataforma y los recursos web educativos integrados para luego evaluar las temáticas propuestas, analizar y comparar los resultados obtenidos entre los dos cursos con contextos educativos diferentes.

\subsection{Encuentro 1}

En la primer reunión se presentó el LMS DotLRN en la sala de cómputo del colegio una vez instalado y configurado el servidor, para dar a conocer su funcionamiento y capacitar al docente y estudiantes sobre las características del curso, los ítems que se trataron fueron:

Acceder a la plataforma, Registro, Iniciar sesión, Observar los cursos que tiene a su cargo, Gestionar de forma básica un curso, Actividades, Foros, Material, Evaluaciones, Calendario, Correo electrónico, Agregar estudiantes a su curso,Introducción al manejo de la plataforma y
Geogebra a sus estudiantes

\subsection{Encuentro 2}

Con el docente de la asignatura de geometria que se orienta en los grados noveno A y noveno $\mathrm{B}$, con 70 estudiantes, se realiza las siguientes actividades:

Capacitación para enseñar a estudiantes su registro en la plataforma; Asignación de estudiantes a su respectivo curso; Se profundizó la capacitación de la administración del curso en la plataforma. Agregar el applet del servicio e-learning al curso; Familiarización del docente con el servicio e-learning para enseñar a sus estudiantes.

\subsection{Encuentro 3.}

Para esta jornada el grado noveno A, recibe la explicación del tema haciendo uso del contexto b-learning, conformado por la plataforma, Geogebra, computadores, wifi y video beam. El grado Noveno $\mathrm{B}$ hace su proceso pedagógico convencional. A los estudiantes les explicó como ingresar a la plataforma y solicitar una cuenta de usuario; El administrador habilita las cuentas con privilegios de estudiante; El administrador asigna los estudiantes como miembros de su curso; Los 
estudiantes ingresan con su respectivo login y contraseña a la plataforma; El docente explica la temática de clase y como ingresar al curso, dando una introducían general de la plataforma; El docente da la introducción a Geogebra que esta integrado en la plataforma.

El docente indica cómo acceder al recurso web (geogebra) y realiza una actividades didácticas, tales como: consultar material digital, solucionar el taller mediante el uso del foro de la plataforma y explica como ingresar y desarrollar la evaluación propuesta.

\subsection{Encuentro 4.}

En este encuentro se hacen las evaluaciones de los tema explicado en los dos cursos, (9A y 9B), la prueba se hace con base a las competencias que se requieren. Se obtienen los resultados tabulados en la tabla 3. Para esta comparación se tienen en cuenta el numero de estudiantes que ganaron y perdieron en los dos cursos; en una calificación de 0 a 5 , la evaluación se pierde con un puntaje menor a 3,0 .

Tabla 2. Verificación de criterios de integración para geogebra

\begin{tabular}{|c|c|c|c|c|}
\hline \multirow{2}{*}{$\begin{array}{c}\text { Contexto de } \\
\text { aprendizaje }\end{array}$} & \multicolumn{2}{|c|}{ \# de Estudiantes en rango de evaluación } & \multicolumn{2}{|c|}{ Análisis porcentual de rendimiento } \\
\cline { 2 - 5 } & Perdieron & Ganaron & Perdieron & Ganaron \\
\hline 9A (B-Learning) 2 & & 33 & $5,7 \%$ & $94,3 \%$ \\
\hline 9B (Convencional) & 14 & 21 & $40 \%$ & $60 \%$ \\
\hline
\end{tabular}

\subsection{Encuentro 5.}

En el grupo que usaron el contexto b-learning solo el 5,7 \% presentaron un rendimiento bajo mientras los que no lo usaron el $40 \%$. En el grupo que usaron el contexto b-learning el 94,3\% ganaron la prueba mientras los que no la usaron el $60 \%$. Con el uso de herramientas TIC en este caso de estudio se muestra que de 35 estudiantes, 12 estudiantes de mas lograron superar la prueba, esto equivale a reducir en un $34 \%$ la perdida de la temática.

\section{Conclusiones}

Con la aplicación de una de las estrategias de aprendizaje usadas en la educación superior, como lo es el b-learnig en la adquisición de competencias de geometría en la educación media demuestra:

El uso del b-learning junto al LMS con el framework propuesto para integrar geogebra como herramienta de aprendizaje tiene muy buena acogida en la comunidad educativa por su nivel interactivo.

Se demuestra un mejor rendimiento a los estudiantes que hicieron uso del b-learning respecto a los que solo recibieron la clase de modo convencional.

Como trabajo futuro se deja planteado llevar este tipo de casos en contextos MOOC y en diferentes áreas del conocimiento

\section{Referencias}

1].Sotelo, F., Solarte, M.: Marco de Referencia para la Integración de Recursos Web como Servicios de E-Learning en .LRN. Universidad del Cauca, Popayán, Tesis de Maestría en Ingeniería Telemática, (2013)

[2] Cabero, J.: Bases pedagógicas del e-learning. Revista de Universidad y Sociedad del Conocimiento (RUSC), vol. 3, no. 1. (2006).

[3] Boneu, J.: Plataformas de e-learning para el soporte de contenidos educativos abiertos. Revista de Universidad y Sociedad del Conocimiento (RUSC),(2007).

[4] Bartolomé, A.:Blended Learning. Conceptos básicos.Píxel-Bit. Revista de Medios y Educación, pp. 7-20, (2004). Disponible en: http://www.lmi.ub.es/personal/bartolome/ 
articuloshtml/04_blended_learning/

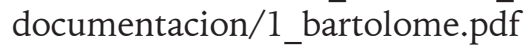

[5] Sotelo, F., Ordóñez, A., Solarte, M.: Marco de referencia para la integración de recursos Web como servicios de e-learning en .LRN. Revista Tecnura, 19(46), 79-91. doi:10.14483/udistrital.jour. tecnura.2015.4.a06. (2015).

[6] Sotelo, F., Ordóñez, A.: Evaluación del marco de referencia para la integración de recursos Web en DotLRN, 10CCC - 10 Congreso Colombiano de Computación, Bogotá Colombia, Septiembre 9 (2015).

[7] Institución Educativa Rafael Pombo: Manual de convivencia institucional Documento, en construcción (2017). 